\title{
Occupational Exposure to Blood and Bodily Fluids among Health Care Work- ers in a Medical Teaching Hospital
}

\author{
Alhazmi RA ${ }^{* 1}$ and Surber SJ $^{2}$ \\ ${ }^{1}$ Assistant Professor, Prince Sultan Bin Abdulaziz College for EMS, King Saud University \\ ${ }^{2}$ Research Fellow, West Virginia University, Morgantown, West Virginia, USA
}

*Corresponding author: Alhazmi RA, EMS Department, Prince Sultan Bin AbdulAziz College for EMS, King Saud University, PO Box 25063, Riyadh, Saudi Arabia, 11466, Tel: +966-560231313, E-mail: rialhazmi@ksu.edu.sa

Citation: Alhazmi RA, Surber SJ (2018) Occupational Exposure to Blood and Bodily Fluids among Health Care Workers in a Medical Teaching Hospital. J Public Health Hygiene Safety 1(1): 101. doi: 10.15744/2767-8792.1.101

Received Date: March 21, 2018 Accepted Date: December 17, 2019 Published Date: December 19, 2019

\begin{abstract}
The objective of this study was to evaluate blood and bodily fluids exposure through needlestick injuries (NSI) and non-percutaneous incidents among healthcare workers (HCWs). This project utilized a dataset collected from J. W. Ruby Memorial Hospital in Morgantown, West Virginia, between January 1, 2014 and August 15, 2017. Data included de-identified codes of employees, occupations, types of exposure to bloodborne pathogens, routes of exposure, infectious disease exposures, and time incidents. During this time period, 655 incident reports were documented and finalized in regards to blood or body fluid exposure. HCWs had 506 (77.25\%) NSIs and 149 (22.74\%) non-percutaneous incidents. The majority of the HCWs (331,50.53\%) were nurses who were occupationally exposed, with 239 (47.23\%) incidents reporting NSIs and 92 (61.74\%) incidents reporting non-percutaneous exposures. Chi-square tests were used, and there was a statistically significant association between occupations and exposure incidents ( $\mathrm{P}$-value $\mathrm{p}=<.0001$ ). Occupations and shift time were statistically associated with the routes of exposure $(\mathrm{p}=<.0001)$. NSIs had higher incidents than non-percutaneous exposures. Exposure to bloodborne pathogens largely occurred among nurses and physicians. Future research should assess the type and duties of nurses and physicians, as well as examine differences in the characteristics of HCWs regarding of the characteristics of shifts (such as time and length) which lead to NSIs
\end{abstract}

Keywords: Healthcare; Exposure; Needlestick; Hospital; Infection Control; Hcws

\section{Introduction}

The primary role of healthcare workers (HCWs) is to care for sick and injured patients, which can expose HCWs to a variety of diseases, injuries, and conditions. A HCW is any person whose role is directly associated with healthcare, including physicians, nurses, dentists, medical technicians, medical assistants, and students in the healthcare field [1]. Unsurprisingly, HCWs can experience occupational exposure to various forms of hazards including sharps injuries, harmful exposures to chemicals and hazardous drugs, back injuries, latex allergy, violence, and stress [2]. HCWs can be exposed to blood or bodily fluids through contact with mucous membranes, non-intact skin contact, or percutaneous injuries. HCWs can face exposures to hepatitis B virus (HBV), hepatitis $\mathrm{C}$ virus (HCV) and human immunodeficiency virus, (HIV), as well as other illnesses transmitted by blood and bodily fluids HCWs are most often exposed to disease through needlestick injuries (NSIs) [3]. NSIs are defined as percutaneous injuries with sharp items contaminated with blood or other bodily fluids [4]. The high level of occupational exposure to blood and bodily fluids among HCWs is more common than in the general population [5-9]. In a 12 year CDC study of selected hospitals in the U.S., 30,945 incident of exposures were reported, with $82 \%$ of those as percutaneous exposures [10]. These exposures most often occurred in in-patient units (36\%) and operating rooms (29\%).Nurses (42\%) were most often exposed, followed by physicians (30\%). Hollow-bore needles (55\%) were involved in the majority of the percutaneous incidents, followed by solid sharps (41\%). Hollow-bore needles carry a higher risk of transmission of bloodborne diseases than other devices. Similar results have been observed elsewhere. One study reported $82.1 \%$ of HCWs experienced percutaneous injuries, and $48.7 \%$ experienced other types of exposure to blood and bodily fluids throughout their work in the field [6]. Another study reported 44\% of HCWs experienced NSIs, 31\% had other types of percutaneous injuries, and the rest of the exposures were non-percutaneous [7]. In its review of 13,847 percutaneous incidents at selected U.S. hospitals, the CDC found that $56 \%$ of the injuries were preventable (17\% undetermined), most often because safer devices were available (26\%) or because the needlework was unnecessary (9\%). In $6 \%$ of the cases, safer work practices would have prevented the percutaneous injury. Thus, in the healthcare setting, the high rates of occupational exposures to blood and bodily fluids can be significantly reduced by the use of universal precautions [11]. Methods 
of reducing exposures include using trays to pass sharp items (the neutral zone), recapping devices to prevent needlestick injuries (NSIs), utilizing blunt sutures, and eliminating unnecessary needlework. Failure to comply with universal precautions guidelines significantly increases the rates of exposure, thus endangering HCWs and their patients [6]. Other studies have found hospital environments in which more than half of HCWs did not use protective gloves and also did not wash their hands after having contact with patients [7]. In other study, the HCWs knowledge and training regarding different types of infectious diseases and universal precautions compliance were not at an acceptable level, with approximately more than two-thirds of participants reported that they had not been trained on the prevention of bloodborne pathogens and the risks of occupational exposure [12]. Interventions and safety devices may improve outcomes. Research conducted at a research hospital estimated a rate of 69.0 NSIs per 1,000 fulltime HCWs prior to the introduction of safety devices, which was reduced to 52.4 per 1,000 afterwards [13]. Other research has identified the barriers to overcome with instituting NSI interventions $[13,14,15]$. Some types of HCW occupations may be more susceptible to exposure of bloodborne pathogens [14,16]. A lack of information exists regarding different HCW occupations that are most likely to be exposed to blood and bodily fluids through percutaneous or non-percutaneous exposure. The objective of this study was to evaluate blood and bodily fluids exposure incidents among HWCs at a university research hospital.

\section{Methods}

\section{Study design}

This study utilized a secondary dataset with data that was collected from J. W. Ruby Memorial Hospital in Morgantown, West Virginia, between January 1, 2014 and August 15, 2017. The design was a retrospective HCW record study on the occurrence of blood and bodily fluids exposure incidents amongst HCWs. Employees reported exposures to the Employee Health Clinic of Ruby Memorial Hospital, and all reports were electronically recorded for purposes of employee exposure laws, unrelated to this research. The Employee Health Clinic serves to fulfill the recordkeeping requirements under the Needlestick Safety and Prevention Act and the Bloodborne Pathogens standard for the exposures log. The Act requires healthcare employers to report all sharps injuries and other bloodborne pathogen exposures to the Occupational Safety and Health Administration (OSHA). WVU Healthcare's legally-required log is maintained in an electronic database, which was provided for this research. No personally identifiable information is available from this database. As part of this research, the Employee Health Clinic provided the dataset in the form of an Excel document, which was de-identified and anonymous. Data included de-identified codes, occupations, types of exposure to bloodborne pathogens, routes of exposure, infectious diseases exposures, and time and date of incidents. Occupations were categorized into major categories, including dental worker, health aide/attendant, laboratory technician, nurse, physician (surgical), technician (surgical) and technician/therapist. Non-healthcare workers were excluded from the analysis. Types of exposures to bloodborne pathogens were categorized into percutaneous injuries and nonpercutaneous exposures. Percutaneous injuries include any exposure through needles, surgical sharp instruments, or glass, and non-percutaneous exposures include any physical exposure to blood and bodily fluids like splashing or touching blood or body fluids. Routes of percutaneous injuries were analyzed, whether transmitted through needles, sharp instruments, or glass, as well as how deep injuries were (superficial, moderate, or deep). Incident data were categorized by year $(2014,2015,2016,2017)$, and incident time was categorized into to three shifts: Shift 1 (8:01 AM-16:00 PM), Shift 2 (16:01 PM-0:00 AM), and Shift 3 (0:01 AM-8:00AM). Infectious disease exposure was analyzed based on the type of disease, including HBV, HCV, and HIV.

\section{Study Sample}

J. W. Ruby Memorial Hospital is a private medical teaching facility and the largest facility in the West Virginia University Medicine family and has over 1,700 employees. [18]. It consists of a 645-bed academic medical center and was the only facility included in this study. The hospital is located in the city of Morgantown, which is in the northern part of West Virginia. As of the 2016 census, the population of the city was 30,855 [19]. The Employee Health Clinic in the hospital is open to all employees, covering annual tuberculosis skin testing, immunizations, eye exams, bloodborne pathogen exposure, flu shots, and lipid panels. The data identified 926 incidents received between January 1, 2014, and August 15, 2017. The occupations were not documented for 263 of the incidents and were excluded from analyses. Non-HCW occupations were identified in 8 of the incidents and were excluded from analyses.

\section{Data Analysis}

Data analysis was performed using Statistical Analysis System (SAS) software, Version 9.4. Descriptive statistics were performed to summarize the characteristics of HCWs regarding the types of exposure and routes of percutaneous injuries. Chi-square tests were used to compare categorical variables. Differences at the $\alpha=0.05$ level were considered statistically significant.

\section{Results}

The data identified 926 incidents received between January 1, 2014, and August 15, 2017, with 913 incidents documented as blood or bodily fluid exposures and 13 documented as other incidents. In 655 incidents occupations were documented as various types of HCWs. Percutaneous injuries occurred in 487 of the 662 incidents. In the final analysis, exposure incidents were primary outcomes, and HCWs occupations were the primary independent variable, so non-HCWs occupations (8 out of 662) and 263 incidents with non-identified occupations were deleted from the analyses. Blood or body fluid exposures were documented and finalized 
in 655 incident reports (Table 1). Percutaneous incidents occurred in 506 (77.25\%) incidents and 149 (22.74\%) incidents were non-percutaneous. The majority of the HCWs (331,50.53\%) were occupationally exposed nurses, with 239 (47.23\%) of the total occupational percutaneous incidents and 92 (61.74\%) of the total non-percutaneous incidents. In this timeframe, nursing incidents were overwhelmingly percutaneous. Of the nurses, 239 (72.20\%) of the 331 nurse-related incidents were percutaneous and 92 (27.79\%). The majority of exposure incidents occurred in 2016 (224, 34.20\%) with 171 (33.79\%) incidents of percutaneous injuries and $53(35.57 \%)$ incidents of non-percutaneous injuries. In addition, the majority of exposure incidents occurred during morning shifts of 8:01 AM-16:00 PM (348, 53.13\%), with 278 (54.94\%) incidents of percutaneous injuries and 70 (46.97\%) incidents of nonpercutaneous injuries. A chi-square test was used to evaluate the relationship between exposure incidents of HCWs and events. There was a statistically significant association only between occupations and exposure incidents $(\mathrm{P}-\mathrm{value}[\mathrm{P}]=<.0001)($ Table 1$)$.

\begin{tabular}{|c|c|c|c|c|}
\hline & \multicolumn{4}{|c|}{ Exposure Incidents } \\
\hline & Non-Percutaneous (\%) & Percutaneous Injuries (\%) & na(\%) & p-value ${ }^{*}$ \\
\hline \multicolumn{5}{|l|}{ Occupation } \\
\hline Dental worker & $0(0.00)$ & $10(1.98)$ & $10(1.52)$ & \\
\hline Health aide/attendant & $9(6.04)$ & $22(4.35)$ & $31(4.73)$ & \\
\hline Laboratory technician & $4(2.68)$ & $24(4.74)$ & $28(4.27)$ & \\
\hline Nurse & $92(61.74)$ & $239(47.23)$ & $331(50.53)$ & \\
\hline Other healthcare occupations & $4(2.68)$ & $5(0.99)$ & $9(1.37)$ & \\
\hline Physician, surgical & $21(14.09)$ & $147(29.05)$ & $168(25.64)$ & \\
\hline Technician, surgical & $4(2.68)$ & $46(9.09)$ & $50(7.63)$ & \\
\hline Technician/therapist, other & $15(10.07)$ & $13(2.57)$ & $28(4.27)$ & $<.0001$ \\
\hline \multicolumn{5}{|l|}{ Year exposure occurred } \\
\hline 2014 & $24(16.11)$ & $55(10.87)$ & $79(12.06)$ & \\
\hline 2015 & $39(26.17)$ & $142(28.06)$ & $181(27.63)$ & \\
\hline 2016 & $53(35.57)$ & $171(33.79)$ & $224(34.20)$ & \\
\hline 2017 (mid-Aug) & $33(22.15)$ & $138(27.27)$ & $171(26.10)$ & 0.25 \\
\hline \multicolumn{5}{|l|}{ Shift exposure occurred (time) } \\
\hline Shift 1 (8:01-16:00) & $70(46.97)$ & $278(54.94)$ & $348(53.13)$ & \\
\hline Shift $2(16: 01-0.00)$ & $45(30.20)$ & $140(27.66)$ & $185(28.24)$ & \\
\hline \multirow[t]{2}{*}{ Shift $3(0.01-8: 00)$} & $34(20.13)$ & $88(17.39)$ & $122(18.62)$ & 0.17 \\
\hline & $149(22.74)$ & $506(77.25)$ & 655 & \\
\hline$n=271$ of 926 reported were del & due to missing value & occupations and as well & the exclusio & on-HCW \\
\hline
\end{tabular}

HCWs identified the routes of percutaneous injuries in 487 incidents, with the causes identified as hollow-bore needles, surgical instruments or other sharp items, or glass (Table 2). In 168 incidents, no routes of incident were identified. The majority of incidents were caused by hollow- bore needles (278, 57.08\%), followed by surgical instruments or other sharp items (199, 40.86\%), and only $10(2.05 \%)$ incidents were caused by glass. The incidents caused by glass had the lowest rate of incidents among all variables. The routes of incidents caused of percutaneous injuries were occupationally driven. Nurses had the highest number of incidents by hollow-bore needles (197, 70.86\%), most likely driven by higher rates of exposures to occupational activities using hollow-bore needles. Surgical physicians were the most likely of the occupations to have percutaneous injuries from surgical instruments or other sharp objects (108,54.27\%). In fact, surgical instruments or other sharp objects caused the most percutaneous injuries of the total 139 injuries of surgical physicians (108,77.70\%). The clinic determined the severity of percutaneous injuries and classified them as deep1, moderate2, or superficial3. None of the injuries reported involved a deep injury. The majority of the percutaneous exposures were superficial injuries $(422,86.67 \%)$, and the majority of those superficial injuries were caused by hollow-bore needles $(234,84.17 \%)$. However, the majority of the moderate injuries were also by hollow-bore needles $(44,15.63 \%)$. Percutaneous injuries during the morning shifts morning shifts (8:01 AM-16:00 PM) were almost evenly split between hollow-bore needles (131, 49.06\%) and surgical instrument or other sharp items (132,49.44\%). Hollow-bore needle incidents were the most common routes of incidents for the afternoon and evening shifts (147,52.88\%). Chi-square tests were used to evaluate the relationship between the routes of incidents caused and characteristics of the incidents. There was a statistically significant association between occupations and the routes of incidents caused ( $\mathrm{p}=<.0001$ ), where hollow-bore needle incidents had the dramatically highest proportion (75.08\%) among other routes, specifically among nurses. In addition, there was a statistically significant association between shift time and the routes of incidents caused, where injuries most likely occurred during morning shifts.

${ }^{1}$ A deep injury is classified as one with more than "some bleeding".

${ }^{2} \mathrm{~A}$ moderate injury is one with punctured skin and "some bleeding".

${ }^{3} \mathrm{~A}$ superficial injury is one with "little or no bleeding". 


\begin{tabular}{|c|c|c|c|c|c|}
\hline & \multicolumn{5}{|c|}{ Routes of Incident Caused } \\
\hline & $\begin{array}{l}\text { HOLLOW-BORE } \\
\text { NEEDLE (\%) }\end{array}$ & $\begin{array}{c}\text { SURGICAL INSTRUMENT OR } \\
\text { OTHER SHARP ITEM (\%) }\end{array}$ & $\begin{array}{l}\text { GLASS } \\
(\%)^{*}\end{array}$ & na & P-value \\
\hline \multicolumn{6}{|l|}{ Occupation } \\
\hline Dental worker & $3(1.08)$ & $7(3.25)$ & $0(0.00)$ & 10 & \\
\hline Health aide/attendant & 11(3.96) & $8(4.02)$ & $0(0.00)$ & 19 & \\
\hline Laboratory technician & $23(8.27)$ & $0(0.00)$ & $0(0.00)$ & 23 & \\
\hline Nurse & 197(70.86) & $33(16.58)$ & $4(40.0)$ & 234 & \\
\hline Other healthcare occupations & $2(0.72)$ & $3(1.51)$ & $0(0.00)$ & 5 & \\
\hline Physician, surgical & $30(10.79)$ & $108(54.27)$ & $1(10.0)$ & 139 & \\
\hline Technician, surgical & $8(2.88)$ & $36(18.09)$ & $2(20.0)$ & 46 & \\
\hline Technician/therapist, other & $4(1.44)$ & $4(2.01)$ & $3(30.0)$ & 11 & $<.0001$ \\
\hline \multicolumn{6}{|l|}{ Year exposure occurred } \\
\hline 2014 & $32(11.51)$ & $20(10.05)$ & $1(10.0)$ & 53 & \\
\hline 2015 & $83(29.86))$ & $47(23.62)$ & $4(40.0)$ & 134 & \\
\hline 2016 & $97(34.89)$ & $66(33.17)$ & $4(40.0)$ & 167 & \\
\hline 2017 (mid-Aug) & $66(23.74)$ & $66(33.17)$ & $1(10.0)$ & 133 & 0.27 \\
\hline \multicolumn{6}{|l|}{ Shift exposure occurred } \\
\hline Shift 1 (8:01-16:00) & $131(47.12)$ & $132(66.33)$ & $4(40.00)$ & 267 & \\
\hline Shift 2 (16:01-0.00) & $84(30.22)$ & $49(24.62)$ & $1(10.00)$ & 134 & \\
\hline Shift 3 (0.01-8:00) & $63(22.66)$ & $18(9.05)$ & $5(50.00)$ & 86 & $<.0001$ \\
\hline \multicolumn{6}{|l|}{ Injury Depth } \\
\hline Superficial & $234(84.17)$ & 179(89.95) & $9(90.0)$ & 422 & \\
\hline \multirow[t]{2}{*}{ Moderateb } & $44(15.63)$ & $20(10.05)$ & $1(10.0)$ & 65 & 0.17 \\
\hline & $278(57.08)$ & 199 (40.86) & $10(2.05)$ & 487 & \\
\hline
\end{tabular}

TABLE 2: Demographic information of HealthCare Workers and relationships with types of percutaneous injuries. (n=487)

524 incidents involved exposure to infectious diseases such as HBV and HCVs (Table 3). 591 (94.65\%) incidents were exposed to $\mathrm{HBV}$, and $5.44 \%$ were not exposed to HBV (Table 3). The exposure to HBV was relatively similar between non-percutaneous and percutaneous incidents, with $90.03 \%$ and $95.72 \%$, respectively. 517(82.72\%) incidents were exposed to HCV, and $17.28 \%$ were not exposed to HCV (Table 3). However, the exposure to HCV had a quite similar proportion between non-percutaneous and percutaneous incidents, with $23.13 \%$ and $15.68 \%$, respectively. Accordingly, there was a statistically significant association between HCV exposure and exposure incidents $(\mathrm{p}=0.04)$.

\begin{tabular}{|c|c|c|c|}
\hline Exposure $^{*}$ & Non-Percutaneous (\%) & Percutaneous (\%) & $\mathbf{n}(\%)$ \\
\hline \multicolumn{2}{|c|}{ Hepatitis B Exposure } & & \\
\hline Yes & $121(90.03)$ & $470(95.72)$ & $591(94.65)$ \\
\hline No & $13(9.30)$ & $21(4.25)$ & $34(5.44)$ \\
\hline \multicolumn{2}{|c|}{ Hepatitis C Exposure } & $77(15.68)$ & $108(17.28)$ \\
\hline Yes & $31(23.13)$ & $414(84.32)$ & $517(82.72)$ \\
\hline No & $103(78.87)$ & 491 & 625 \\
\hline \multicolumn{2}{|c|}{134} & was therefore deleted from analysis \\
\hline “HIV test indication was not included due to low sample size (36) and \\
\hline
\end{tabular}

TABLE 3: Healthcare Workers Reporting Occupational Exposure to Infectious Diseases ( $\mathrm{n}=625)$

\section{Discussion}

HCWs treat a plethora of medical conditions, and therefore, face occupational exposure to a variety of infectious diseases. Because of the risks of disease, the Centers for Disease Control and Prevention (CDC) and the United States Occupational Safety and Health Administration (OSHA) created universal precautions and guidelines for bloodborne pathogen exposure and needlestick safety guidelines [20]. However, exposures still occur, and it is critical for the health of HCWs, patients, and the general public to understand what exposure issues still continue to exist. This study evaluated the occupational exposure incidents among HWCs 
through blood and bodily fluids in a university research hospital setting. This study identified the occurances of exposure to blood pathogens and bodily fluids through percutaneous and non-percutaneous exposure among HCWs in order to assess possible approaches to prevent such incidents. Research has shown that occupational exposure to blood and bodily fluids continues to exist among HCWs [10-14]. Our research finds similar incidents in a research university hospital reported to that hospital's employee clinic. In the time period of January 1,2014 and August 15,2017, different occupations of HCWs reported 655 incidents regarding blood and bodily fluids exposure from in a university research hospital. Of those exposures, HCWs reported, the majority were percutaneous injuries $(n=506,77.25 \%)$, and $22.74 \%(n=149)$ reported non-percutaneous exposure to blood and bodily fluids (Table 1). Previous studies support our findings that exposures occur due to percutaneous injuries more than non-percutaneous exposures [21-23]. Occupations were associated with the occurrence of percutaneous injuries, which is consistent with past studies $[24,25]$. As various studies have identified, exposure to bloodborne pathogens predominantly occur among nurses, physicians, and lab workers [24,25]. However, our study found that nurses (50.53\%) were exposed to blood and bodily fluids more than other HCW occupations, followed by surgical physicians (25.64\%). This is likely due to higher rates and durations of exposure. Nurses make contact with patients for longer periods of time compared to other occupations, which consequently exposes to blood and bodily fluids. It is estimated that $70.4 \%$ of registered nurses (RNs) experience at least one needle stick injury per year, while the less experiences nurses received more needlestick injuries [26]. Published studies reveal most nurses inadequately comply with universal precautions worldwide, where nurses are selective regarding the implementation of universal precautions recommendations, which is strong evidence of the low level of compliance of universal precautions [27,28]. In our study, we included all types of nurses in one category. Future studies should assess the extent of the exposure among different types of nurses in order to find out the better preventive measures. The majority of percutaneous injuries incidents were caused by hollowbore needles (75.08\%), followed by surgical or other sharp items (40.86\%). Previous studies are consistent with our study, as NSIs, particularly with hollow-bore needles, are the most frequent items resulting in exposure to blood and bodily fluids [29,30]. Percutaneous injuries caused by hollow-bore needles were the most frequent incidents among nurses (70.86\%), whereas surgical instruments or other sharp items were the most frequent incidents among physicians (surgical) (54.27\%). In addition, shift times when injuries occurred were positively associated with percutaneous injuries, where morning shifts comprised a greater percentage of injures among HCWs. Previous research showed the majority of exposures occurred during the morning shift [31]. This may be attributed to busy schedules and greater demands of patients during morning shifts. However, there are previous studies inconsistent with our results, which found that fatigue and sleep deprivation affect extended shifts more than regular working hours, so the night shift is associated with high percentages of percutaneous injuries among HCWs [32,33]. Future research should examine differences between the characteristics of HCWs regarding types of shifts resulting in percutaneous injuries. HCWs encounter all type of infectious diseases during performance their duties, resulting in concerns regarding transmission of blood borne pathogens. In United States, cases of acute hepatitis B increased by $20.7 \%$ to 3,370 cases in 2015 in general population, and cases of acute HCV infection increased by increased 11\% to 2,436 cases in 2015 [34]. In our study, most reported incidents are associated with patients who have hepatitis B, C or both. More protective procedures should be applied through attending Bloodborne pathogen education and implementing standard precautions compliance. Future study should assess the extent of HCWs' knowledge about infectious diseases, its routes of transmission and its effects and explore the extent of HCWs concern to consistently attend continuing medical education programs. This study has some limitations, underreporting of blood and bodily fluids exposure is missed in our data, resulting in an underestimate of the true magnitude of the problem. For example, some HCWs who were exposed tended to report, and others did not report the incidents because they might believe the exposure was not significant. Therefore, these results cannot necessarily be generalized, as the results underestimate the problem. Moreover, the dataset lacks information, including gender, years of experience, shift schedule, and blood pathogen training attendance. These information can help to assess different characteristics of HCWs and also estimate the true burden of the exposure among HCWs.

\section{Conclusion}

Exposure to blood and body fluids is a concern among HCWs. The majority of incidents reported here were percutaneous injuries. Exposure to bloodborne pathogens largely occur among nurses and physicians. Future studies should assess all types and duties of nurses and physicians in order to estimate the magnitude of the exposure to help establish better preventive measures based on the workplace environment. The majority of percutaneous injuries incidents were caused by hollow-bore needles, followed by other sharp items. Additionally, morning shifts comprised a greater percentage of percutaneous injuries among HCWs. Future research should examine differences based on characteristics of HCWs regarding types of shifts resulting in percutaneous injuries. The exposure to hepatitis B and C had more reporting among HCWs. Future study should assess the extent of HCWs' knowledge about infectious diseases, its routes of transmission and its effects and explore the extent of HCWs concern to consistently attend continuing medical education programs

\section{Declaration of Interest}

The authors report no conflicts of interest. The author alone is responsible for the content and writing of the paper.

\section{Acknowledgement}

We would like to thank Darlene Davisson and other members of Employee Health at West Virginia University Medicine for their effort and support to provide the dataset. I would also thank Dr Parker David for his assistance for using the data. 


\section{References}

1. Kuhar DT, Henderson DK, Struble KA, Heneine W, Thomas V et al. (2013) Updated US public health service guidelines for the management of occupational exposures to human immunodeficiency virus and recommendations for postexposure prophylaxis. Infect Control Hosp Epidemiol 34: 875-92.

2. Centers for Disease Control and Prevention (CDC) (2017) Healthcare Workers. The National Institute for Occupational Safety and Health (NIOSH).

3. Nienhaus A, Kesavachandran C, Wendeler D, Haamann F, Dulon M (2012) Infectious diseases in healthcare workers-an analysis of the standardised data set of a German compensation board. J J Occup Med Toxicol 7: 8.

4. Centers for Disease Control and Prevention (CDC) (2013). Stop Sticks Campaign. The National Institute for Occupational Safety and Health (NIOSH).

5. Zhang M, Wang H, Miao J, Du X, Li T, et al. (2009) Occupational exposure to blood and body fluids among health care workers in a general hospital, China. Am J Ind Med 52: 89-98.

6. Liu X, Sun X, van Genugten L, Shi Y, Wang Y, et al. (2014) Occupational exposure to blood and compliance with standard precautions among health care workers in Beijing, China. Am J Infect Control 42: 37-8.

7. Sabermoghaddam M, Sarbaz M, Lashkardoost H, Kaviani A, Eslami S, et al. (2015) Incidence of occupational exposure to blood and body fluids and measures taken by health care workers before and after exposure in regional hospitals of a developing country: A multicenter study. Am J Infect Control 43 : 1137.

8. Hajjaji DM, Chaabouni T, Jmal HK, Messadi AF, Abdennadher M, et al. (2014) Occupational blood exposure among health care personnel and hospital trainees. Int J Occup Environ Med 5: 57-61.

9. Coppola N, De Pascalis S, Onorato L, Calò F, Sagnelli C, et al. (2016) Hepatitis B virus and hepatitis C virus infection in healthcare workers. Wrl J Hep 8: 273.

10. The National Surveillance System for Healthcare Workers (NaSH) Summary Report for Blood and Body Fluid Exposure (1995-2007).

11. Hamlyn E, Easterbrook P (2007) Occupational exposure to HIV and the use of post-exposure prophylaxis. Occup Med 57: 329-36.

12. Hosoglu S, Akalin S, Sunbul M, Otkun M, Ozturk R (2011) Healthcare workers' compliance with universal precautions in turkey. Med Hyp 77: 1079-82.

13. Hoffmann C, Buchholz L, Schnitzler P (2013) Reduction of needlestick injuries in healthcare personnel at a university hospital using safety devices. J Occup Med Toxicol 8: 20.

14. Brasel KJ, Mol C, Kolker A, Weigelt JA (2007) Needlesticks and surgical residents: who is most at risk. Journal of surgical education 64: 395-8.

15. Tarigan LH, Cifuentes M, Quinn M, Kriebel D (2015) Prevention of needle-stick injuries in healthcare facilities: a meta-analysis. Infect Control Hosp Epidemiol 36: 823-9.

16. Patrician PA, Pryor E, Fridman M, Loan L (2011) Needlestick injuries among nursing staff: association with shift-level staffing. Am J Infect Control 39: 477-82. 17. Choi LY, Torres R, Syed S, Boyle S, Ata A, et al. (2017) Sharps and needlestick injuries among medical students, surgical residents, faculty, and operating room staff at a single academic institution. J Surg Educ 74: 131-6.

18. WVU Medicine (2017) JW Ruby Memorial Hospital WVU Physicians. Retrieved October 2, 2017. from http://wvumedicine.org/ruby-memorial-hospital/ about-us/

19. United States Census Bureau (2016) Quick Facts, Morgantown City, West Virginia. Retrieved October 7, 2017. from https://www.census.gov/quickfacts/fact/ table/morgantowncitywestvirginia/PST045216

20. Centers for Disease Control and Prevention. Recommendation for prevention of HIV Transmission in Health-care settings. Morbidity and Mortality Weekly Report 36: 1-18. Retrieved from https://www.cdc.gov/mmwr/preview/mmwrhtml/00023587.htm

21. Deuffic-Burban S, Delarocque-Astagneau E, Abiteboul D, Bouvet E, Yazdanpanah Y (2011) Blood-borne viruses in health care workers: prevention and management. J Clin Virol 52: 4-10.

22. Gönen I, Geyik MF (2011) Percutaneous injuries among healthcare workers at a general hospital. Journal of Microbiology and Infectious Diseases 1: 26-30.

23. Mbaisi EM, Wanzala P, Omolo J, (2013) Prevalence and factors associated with percutaneous injuries and splash exposures among health-care workers in a provincial hospital, Kenya, 2010. Pan Afr Med J 14: 10.

24. Pérez-Diaz C, Calixto OJ, Faccini-Martínez ÁA, Bravo-Ojeda JS, Botero-García CA, et al. (2015) Occupational exposure to blood borne pathogens among healthcare workers: A cross-sectional study of a registry in Colombia. J Occup Med Toxicol 10: 45.

25. Marziale MHP, Rocha FLR, Robazzi MLDCC, Cenzi CM, Santos HECD, Trovó MEM (2013) Organizational influence on the occurrence of work accidents involving exposure to biological material. Revista latino-americana de enfermagem, 21:199-206.

26. Cho E, Lee H, Choi M, Park SH, Yoo IY, Aiken LH (2013) Factors associated with needlestick and sharp injuries among hospital nurses: A cross-sectional questionnaire survey. Int J Nurs Stud 50: 1025-32.

27. Efstathiou G, Papastavrou E, Raftopoulos V, Merkouris A (2011) Compliance of cypriot nurses with standard precautions to avoid exposure to pathogens. Nurs Health Sci 13: 53-9.

28. Pereira FMV, Lam SC, Chan JHM, Malaguti-Toffano SE, Gir E (2015) Difference in compliance with standard precautions by nursing staff in Brazil versus Hong Kong. Am J Infect Control 43: 769.

29. Martins A, Coelho AC, Vieira M, Matos M, Pinto ML (2012) Age and years in practice as factors associated with needlestick and sharps injuries among health care workers in a Portuguese hospital. Accid Anal Prev 47: 11-5.

30. Kakizaki M, Ikeda N, Ali M, Enkhtuya B, Tsolmon M, et al. (2011) Needlestick and sharps injuries among health care workers at public tertiary hospitals in an urban community in Mongolia. BMC Research Notes 4: 184.

31. Chalya PL, Seni J, Mushi MF, Mirambo MM, Jaka H, et al. (2015) Needle-stick injuries and splash exposures among health-care workers at a tertiary care hospital in north-western Tanzania. Tanzania J Health Res 17.

32. Wicker S, Ludwig AM, Gottschalk R, Rabenau HF (2008) Needlestick injuries among health care workers: Occupational hazard or avoidable hazard. Wien Klin Wochenschr 120: 486-92.

33. Dembe AE, Delbos R, Erickson JB (2009) Estimates of injury risks for healthcare personnel working night shifts and long hours. Qual Saf Health Care 18: $336-40$.

34. Centers for Disease Control and Prevention (CDC) (2015) Surveillance for Viral Hepatitis-United States 2015. 


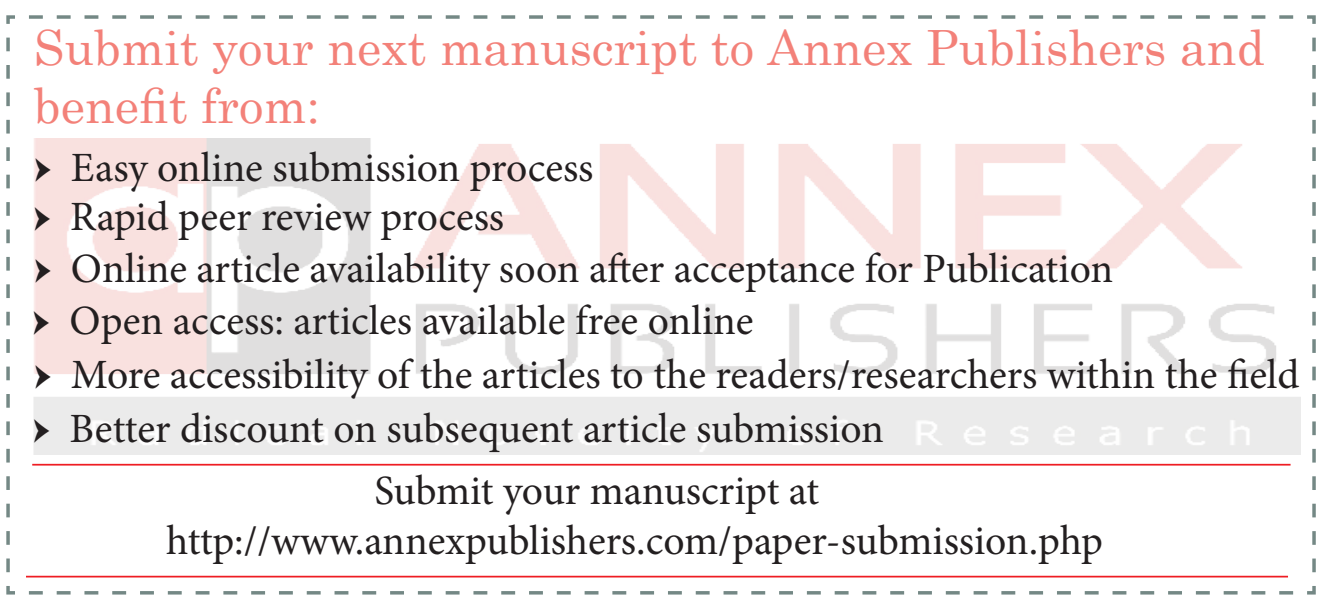

